\title{
The Case of Pyelonephritis and Renal Cyst Infection Diagnosed by Diffusion-Weighted Magnetic Resonance Imaging
}

\author{
Hiroki Adachi ${ }^{\mathrm{a}}$, Akahito Sako ${ }^{\mathrm{a}, \mathrm{d}}$, Ayako Ikuta ${ }^{\mathrm{b}}$, Kazuha Nakamura ${ }^{\mathrm{b}}$, Akihiro Machitoric, \\ Shuichi Mishima ${ }^{a}$, Hidekatsu Yanai ${ }^{\mathrm{a}}$
}

\begin{abstract}
Diffusion-weighted magnetic resonance imaging (DWMRI) is widely used for the diagnosis of cerebrovascular diseases. However, it is recently gaining attention for the diagnosis of infection and tumor in the abdominal region. We experienced a case of an 80 -year-old woman whose diagnosis was difficult to differentiate between pyelonephritis and cholangitis. DWMRI showed high signal intensity in renal parenchyma and renal cyst, which led to the diagnosis of pyelonephritis and renal cyst infection. Radiation exposure and contrast media could be avoided for DWMRI. DWMRI is a safe and reliable modality in diagnosing pyelonephritis and renal cyst infection.
\end{abstract}

Keywords: Diffusion-weighted magnetic resonance imaging; Pyelonephritis; Urinary tract infection; Renal cyst infection

\section{Introduction}

Urinary tract infection (UTI) can be a difficult disease to diagnose, because clinical symptoms, physical examination, urinalysis, ultrasonography and computed tomography (CT) sometimes show no specific findings for UTI. Diffusionweighted magnetic resonance imaging (DWMRI) has been used for cerebral diseases. However, it is recently used for

\footnotetext{
Manuscript accepted for publication May 16, 2014

${ }^{a}$ Department of Internal Medicine, Kohnodai Hospital, National Center for Global Health and Medicine, 1-7-1 Kohnodai, Ichikawa, Chiba, 272-8516, Japan

bational Center for Global Health and Medicine, 1-21-1 Toyama, Shinjuku, Tokyo, 162-8655, Japan

${ }^{\mathrm{c}}$ Department of Radiology, Kohnodai Hospital, National Center for Global Health and Medicine, 1-7-1 Kohnodai, Ichikawa, Chiba, 272-8516, Japan

${ }^{\mathrm{d}}$ Corresponding Author: Akahito Sako, Department of Internal Medicine, Kohnodai Hospital, National Center for Global Health and Medicine, 1-7-1 Kohnodai, Ichikawa, Chiba, 272-8516, Japan.

Email: dsako@hospk.ncgm.go.jp
}

doi: http://dx.doi.org/10.14740/wjnu170e the detection of the abdominal infection and malignant diseases in clinical practice. Here, we report a case of pyelonephritis and renal cyst infection diagnosed by DWMRI.

\section{Case Report}

An 80-year-old female experienced nausea and vomiting, which was followed by malaise, and visited the emergency room at our hospital. She had dermatomyositis and type 2 diabetes mellitus. Dermatomyositis was stable by taking 5 $\mathrm{mg}$ of predonisolone. She was also taking glimepiride for diabetes, and $\mathrm{HbAlc}$ was $7.0 \%$.

On admission, body temperature was $35.6{ }^{\circ} \mathrm{C}$, and other vital signs were normal. She did not show fever before admission. Physical examination did not show abnormal signs of the abdomen and costovertebral angle tenderness. Blood examination demonstrated elevation of aspartate aminotransferase (AST) at $80 \mathrm{U} / \mathrm{L}$, alanine aminotransferase (ALT) $47 \mathrm{U} / \mathrm{L}$, alkaline phosphatase (ALP) $553 \mathrm{IU} / \mathrm{L}$ and $\gamma$-glutamyltranspeptitase ( $\gamma$-GTP) $140 \mathrm{IU} / \mathrm{L}$. Total bilirubin $(0.5 \mathrm{mg} / \mathrm{dL})$ was within normal limit. Leucocyte count was $13.5 \times 10^{9} / \mathrm{L}$, and C-reactive protein was $26.8 \mathrm{mg} / \mathrm{dL}$, which was remarkably high. Urinalysis showed slightly elevated number of leukocyte, which was 5 - 9 per high power field. Ultrasonography demonstrated enlargement of the gallbladder and dilatation of the bile duct, but the gallbladder wall was not thick. Right renal pelvis was slightly dilated, and right renal cortex seemed edematous. Contrast-enhanced CT also showed enlargement of the gallbladder and dilatation of the bile duct, but did not reveal any biliary stone or tumor that could have caused obstruction. CT also showed slightly swollen right kidney. It was difficult to differentiate UTI and cholangitis. If the diagnosis was cholangitis, biliary drainage was necessary. We first started to use sulbactam/ampicillin 3 g every $8 \mathrm{~h}$ to treat both possible diseases. DWMRI exhibited high signal intensity, and apparent diffusion coefficient (ADC) showed low signal intensity on the right renal pelvis compared with the left one (Fig. 1a, b), suggesting the existence of pyelonephritis. DWMRI also revealed high intensity in one of the cysts in the right kidney, which indicated renal cyst infection (Fig. 1c). After a few days, E. coli was isolated 

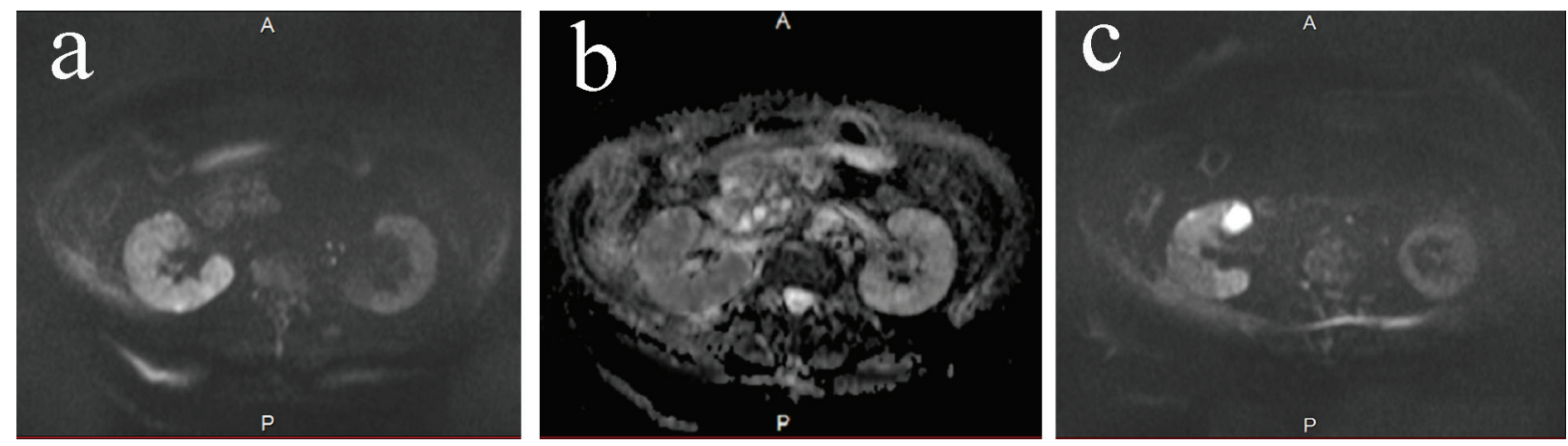

Figure 1. (a) DWMRI on day 0 . The high signal intensity was seen in the right kidney. The intensity was apparently higher than the left kidney. (b) ADC map on day 0 . The intensity was low in the right kidney. (c) DWMRI on day 0 . One of the cyst showed high intensity.

in culture of the blood and urine, and the urinary bacteria were $10^{4}$ colony forming unit $(\mathrm{CFU}) / \mathrm{mL}$. We diagnosed as UTI, and changed the antibacterial treatment to cefazolin according to the diagnosis of UTI and drug susceptibility. Cyst drainage was an option, but since she made good progress with the antibiotic treatment alone, we did not conduct cyst drainage. Serum levels of AST, ALT, ALP and $\gamma$-GTP decreased, and bile duct dilatation disappeared, and then she was discharged from the hospital. DWMRI was performed over two times for follow-up, which showed low intensity of the parenchyma and cyst, suggesting that the pyelonephritis and cyst infection was well treated with antibiotics (Fig. 2). DWMRI after the treatment demonstrated significantly reduced intensity of renal parenchyma and cyst, confirming the usefulness of DWMRI for diagnosis of pyelonephritis and renal cyst infection.

\section{Discussion}

UTI is the most frequent infection in geriatric patients, but the diagnosis is difficult due to high prevalence of asymptomatic bacteriuria. Asymptomatic bacteriuria is observed in
$30 \%$ of male and $50 \%$ of female among 80 years of age or more [1]. Diagnosis of UTI is made with various findings, including clinical history, physical examination, urinalysis, urine culture and imaging. The British Medical Research Council Bacteriuria Committee defined acute pyelonephritis as a syndrome consisting of loin pain, tenderness and pyrexia accompanied by bacteriuria, bacteremia, pyuria and sometimes hematuria [2]. Elderly patients frequently lack typical symptoms, and the association between urinary symptoms and a urinary infection is not clear. A systematic review showed that one or more symptoms of UTI, which include dysuria, frequent urination, hematuria and back pain, or costovertebral angle tenderness, increase the probability of UTI in woman to approximately $50 \%$ [3]. The reference standard for diagnosing UTI is the presence of "significant" bacteriuria, defined as the isolation of at least $10^{5} \mathrm{CFU} / \mathrm{mL}$ [3]. Some reports suggested that the minimum threshold of bacteriuria varies from $10^{2}$ to $10^{5}$ for the diagnosis of UTI $[4,5]$. But this diagnosis of bacteriuria also includes patient with asymptomatic bacteriuria, which has no need for antibiotic therapies. Contrast-enhanced CT is used for the evaluation of patients with acute pyelonephritis. CT can detect focal parenchymal abnormalities and perinephric fluid collection and
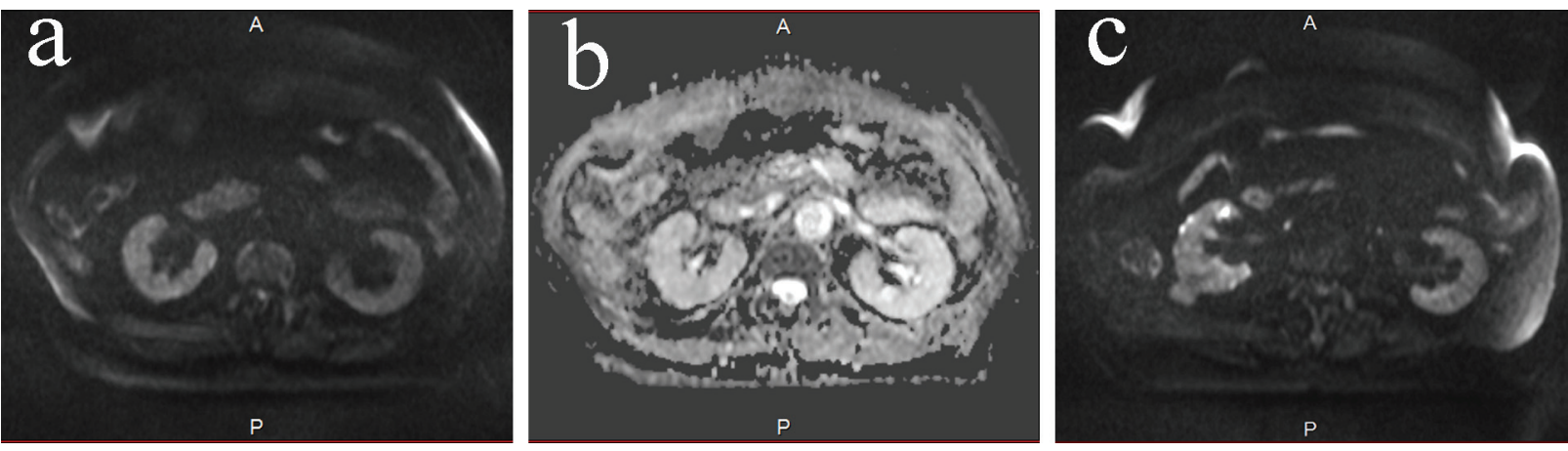

Figure 2. (a) DWMRI on day 58. The signal intensity in the right kidney lowered, which was obvious comparing to Figure 1a. The intensity was same between the right and the left kidney. (b) ADC map on day 58. Right and left kidney had the same intensity. (c) DWMRI on day 58. The intensity of the infected cyst had decreased. 
abscess [6]. Ultrasound is relatively less sensitive compared with contrast-enhanced $\mathrm{CT}$ in the evaluation of pyelonephritis. Ultrasound can miss subtle changes of pyelonephritis, and often underestimates the severity of pyelonephritis [6].

Our case was difficult to differentiate between pyelonephritis and cholangitis, because several examinations suggested the possibility of cholangitis, such as the elevation of serum levels of AST, ALT, ALP, $\gamma$-GTP, and the existence of the dilatation of the bile duct by ultrasonography, $\mathrm{CT}$ and MRI. The Tokyo Guideline for the management of acute cholangitis presents the diagnostic criteria for acute cholangitis which consists of systemic inflammation, cholestasis and biliary dilatation or evidence of the etiology on imaging, such as stricture, stone and stent. According to the guideline, acute cholangitis could have been the diagnosis in our case. Tokyo guideline's sensitivity for the diagnosis for acute cholangitis was $91.8 \%$, and specificity was $77.7 \%$ [7]. Biliary drainage, such as endoscopic retrograde cholangiopancreatography, percutaneous transhepatic cholangiography drainage or open surgical decompression, is important in patient with cholangitis, but since these procedures are invasive, the differential diagnosis of UTI and cholangitis is important. In our case, elevated serum levels of AST, ALT, ALP, $\gamma$-GTP and bile duct dilatation can be explained by cholestasis due to sepsis [8].

DWMRI is used mainly for the diagnosis of neurological diseases, including acute cerebral strokes, intracranial tumors and demyelination diseases [9]. The ADC, as a quantitative parameter calculated from the DWMRI, combines the effects of capillary perfusion and water diffusion in the intracellular extra-vascular space. The use of DWMRI in abdominal diseases is not common, but recently, the application of DWMRI for the diagnosis of abdominal disease, including renal infection, has been reported. DePascale et al underwent DWMRI among 279 patients with acute pyelonephritis. Diagnosis was based on urinalysis or the clinical history. They reported that DWMRI clearly had high sensitivity, specificity, positive/negative predictive value and diagnostic accuracy of acute pyelonephritis, all close to $95 \%$ [10]. Other study showed differences in ADC value among different patient groups, cyst, renal failure, chronic pyelonephritis and hydronephrosis, indicating the usefulness of the ADC values for differentiation of renal lesions [11]. Another study showed signal hyper-intensity of the kidney of acute pyelonephritis and renal abscess on DWMRI as well as hypo-intensity on ADC maps [12]. Ebisu et al showed in vivo imaging of high signal intensity of brain abscess cavity on DWMRI. They also demonstrated that in vitro imaging of aspirated brain abscess fluid showed high signal intensity on DWMRI. Pus is a thick high viscosity fluid consisting of water, inflammatory cells, bacteria, necrotic tissue and proteinaceous exudates. The high signal intensity of DWMRI and low ADC is due to the heavily impeded water mobility caused by the high viscosity and cellularity of pus [13].
DWMRI is also reported to show high intensity of infected cyst $[14,15]$. Our case had both pyelonephritis and cyst infection according to the results of DWMRI. Cyst infection was not detected by ultrasound or CT. MRI also has an important advantage compared to $\mathrm{CT}$, such as the absence of radiation exposure. Since some of the UTI patients are female within their child-bearing age, avoiding radiation exposure is a relevant advantage [16]. Avoiding contrast media is also an advantage, especially in cases with contraindications to administration of iodinated contrast media, such as patients with renal insufficiency [10]. Contrast media is necessary for CT scans, since unenhanced CT is difficult to detect acute bacterial pyelonephritis [6]. Gadolinium-enhanced MRI and DWMRI have a high diagnostic agreement. Gadolinium-enhanced MRI is time-consuming compared to DWMRI [10].

In our case, we were able to differentiate pyelonephritis and renal cyst infection from cholangitis by using DWMRI. To confirm the validity of DWMRI for diagnosis of UTI, further studies, including a large number of patients, are necessary in the future.

In conclusion, DWMRI is a useful modality for the diagnosis of pyelonephritis and renal cyst infection.

\section{Funding}

This work was supported by a grant-in-aid from the National Center for Global Health and Medicine (25-203).

\section{Conflict of Interest}

The authors of this manuscript have nothing to declare.

\section{References}

1. Gavazzi G, Delerce E, Cambau E, Francois P, Corroyer B, de Wazieres B, Fougere B, et al. Diagnostic criteria for urinary tract infection in hospitalized elderly patients over 75 years of age: a multicenter cross-sectional study. Med Mal Infect. 2013;43(5):189-194.

2. Recommended terminology of urinary-tract infection. A report by the members of the Medical Research Council Bacteriuria Committee. Br Med J. 1979;2(6192):717719.

3. Bent S, Nallamothu BK, Simel DL, Fihn SD, Saint S. Does this woman have an acute uncomplicated urinary tract infection? JAMA. 2002;287(20):2701-2710.

4. Stamm WE, Counts GW, Running KR, Fihn S, Turck M, Holmes KK. Diagnosis of coliform infection in acutely dysuric women. N Engl J Med. 1982;307(8):463-468.

5. Giesen LG, Cousins G, Dimitrov BD, van de Laar FA, Fahey T. Predicting acute uncomplicated urinary tract 
infection in women: a systematic review of the diagnostic accuracy of symptoms and signs. BMC Fam Pract. 2010;11:78.

6. Stunell H, Buckley O, Feeney J, Geoghegan T, Browne RF, Torreggiani WC. Imaging of acute pyelonephritis in the adult. Eur Radiol. 2007;17(7):1820-1828.

7. Kiriyama S, Takada T, Strasberg SM, Solomkin JS, Mayumi T, Pitt HA, Gouma DJ, et al. TG13 guidelines for diagnosis and severity grading of acute cholangitis (with videos). J Hepatobiliary Pancreat Sci. 2013;20(1):24-34.

8. Gilroy RK, Mailliard ME, Gollan JL. Gastrointestinal disorders of the critically ill. Cholestasis of sepsis. Best Pract Res Clin Gastroenterol. 2003;17(3):357-367.

9. Chan JH, Tsui EY, Luk SH, Fung SL, Cheung YK, Chan MS, Yuen MK, et al. MR diffusion-weighted imaging of kidney: differentiation between hydronephrosis and pyonephrosis. Clin Imaging. 2001;25(2):110-113.

10. De Pascale A, Piccoli GB, Priola SM, Rognone D, Consiglio V, Garetto I, Rizzo L, et al. Diffusion-weighted magnetic resonance imaging: new perspectives in the diagnostic pathway of non-complicated acute pyelonephritis. Eur Radiol. 2013;23(11):3077-3086.

11. Macarini L, Stoppino LP, Milillo P, Ciuffreda P, Fortunato F, Vinci R. Diffusion-weighted MRI with parallel imaging technique: apparent diffusion coefficient deter- mination in normal kidneys and in nonmalignant renal diseases. Clin Imaging. 2010;34(6):432-440.

12. Verswijvel G, Vandecaveye V, Gelin G, Vandevenne J, Grieten M, Horvath M, Oyen R, et al. Diffusion-weighted MR imaging in the evaluation of renal infection: preliminary results. JBR-BTR. 2002;85(2):100-103.

13. Ebisu T, Tanaka C, Umeda M, Kitamura M, Naruse $\mathrm{S}$, Higuchi T, Ueda S, et al. Discrimination of brain abscess from necrotic or cystic tumors by diffusionweighted echo planar imaging. Magn Reson Imaging. 1996;14(9):1113-1116.

14. Takase Y, Kodama K, Motoi I, Saito K. Cyst infection in unilateral renal cystic disease and the role of diffusion-weighted magnetic resonance imaging. Urology. 2012;80(5):e61-62.

15. Ichioka K, Saito R, Matsui Y, Terai A. Diffusion-weighted magnetic resonance imaging of infected renal cysts in a patient with polycystic kidney disease. Urology. 2007;70(6):1219.

16. Faletti R, Cassinis MC, Fonio P, Grasso A, Battisti G, Bergamasco L, Gandini G. Diffusion-weighted imaging and apparent diffusion coefficient values versus contrast-enhanced MR imaging in the identification and characterisation of acute pyelonephritis. Eur Radiol. 2013;23(12):3501-3508. 